\title{
A whole-team approach to triaging patients: is this the future?
}

\author{
Evaluation of a dental nurse-led triage system in a private dental practice during the COVID-19 pandemic. \\ Br Dent J 2020; 229: https://doi.org/10.1038/s41415-020-2177-3.
}

The COVID-19 pandemic has created a number of unique challenges. Notably on 25 March 2020 we were advised to halt all non-urgent care, remotely triage our patients and provide advice using the 3 As. Patients would then be referred to urgent care centres if required. Practices developed triaging services in numerous ways, however, the practice in this study already had a longstanding out-of-hours nurse-led triage service in place which put them in an ideal position. In medical practice, nurseled triage has been shown to reduce general practitioner contacts by $16 \% .{ }^{1}$ Could nurseled triage also be as effective in general dental practice?

This study covered a triage service in one private practice from 25 March 2020 to 12 June 2020. Calls would be answered by the reception staff and then passed onto the triaging nurse. If support was required, it would be escalated to the dentist on call or the clinical lead for complex situations. Follow up contacts were arranged if required. A separate system was in place for orthodontic emergencies.

Seventy patients were triaged during this period. The most common reasons for contacting the practice were pain, followed by loose extra-coronal restorations and then fractured teeth, crowns or bridges. Of the patients, $68.5 \%$ were managed successfully without input from the dentist. Whereas $25 \%$ of the patients were passed onto a dentist and $5.7 \%$ to the clinical lead. Antimicrobials were only needed for five out of the 70 patients and no patients required referral to an urgent care centre. Sixteen patients called multiple times, and ten of these were managed exclusively by the triaging nurse. Twenty-seven of the patients triaged declined an appointment when offered, for multiple reasons, including the problem resolving, wanting to wait or having shielding family members.

The study shows most patients were managed successfully by the triaging nurse on the first call. There were also no concerns from the dentists about the way patients were managed. The study has shown how a whole-team approach to triaging can work successfully within a practice environment. It would be interesting to see if this system could also work well in practices with different demographics and skill sets, and to get patient feedback. This triaging system could be considered by practices, should it be needed further throughout the pandemic. Could it even be a long-term strategy to provide better access to out-of-hours advice and care?

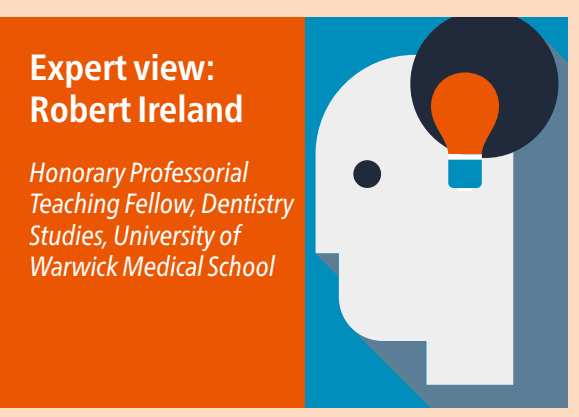

Although this is a limited retrospective study, and therefore the results should be interpreted with caution, this article provides an interesting, relevant and positive example of how the resources of the dental team can be more fully utilised and how the flexibility of a well-trained dental team can adapt efficiently and effectively to a sudden and unexpected change in the provision of emergency dental care.

This study demonstrates how the triage workload can be redistributed $(68 \%$ of patients being managed without dentist input) which supports earlier research demonstrating $85 \%$ agreement between the triage dental nurse and the dentist (of the $15 \%$ where there was disagreement between the dentist and the triage dental nurse, in half the cases the nurse had given a higher triage category than the dentist and in the other half the dentist had given a higher triage category than the nurse). ${ }^{1}$ Dental nurses certainly have the skill set to undertake the role of patient emergency triage subject to having undertaken appropriate training and calibration, bearing in mind that providing 'appropriate patient

\section{Reference}

1. Campbell J L, Fletcher E, Britten $\mathrm{N}$ et al. The Clinical Effectiveness and Cost Effectiveness of Telephone Triage for Managing Same-Day Consultation Requests in General Practice: A Cluster Randomised Controlled Trial Comparing General Practitioner-Led and Nurse-Led Management Systems With Usual Care (The ESTEEM Trial). Health Technol Assess 2015; 19: 1212

By Thomas Turner, Dental Core Trainee 1, The Dudley Group NHS Foundation Trust

advice' is within the scope of practice of the dental nurse but diagnosing is not. COVID19 has forced the reconsideration of the provision of emergency dental care and the triage pro forma forms an important part of the filtering process, of which there are some comprehensive examples of flowcharts available applicable to the COVID-19 pandemic ${ }^{2,3}$ which in this study helped to reduce further appointments by $39 \%$.

It is of concern that some patients were unable to contact their own dentist since the absence of an efficient out-of-hours on-call service is likely to elevate patient stress and potentially negatively impact on a patient's pain threshold, particularly at the time of a pandemic when access to emergency dental care might be restricted.

This limited study opens up the opportunity to consider further research such as exploring the level of patient satisfaction with online triage, the costeffectiveness of utilising dental nurses and the satisfaction of dental nurses broadening their scope of activity within the dental team and their enthusiasm for doing so.

\section{Reference}

1. Trevidy C, Akintola D, Long L, Vooke M W. Dental Nurse triage of patients with dental emergencies Conference: IADR General Session and Exhibition, 2014

2. Scottish Dental Clinical Effectiveness Programme. Management of Acute Dental Problems during the Covid19 Pandemic. 2020. Available at https://www. sdcep.org.uk/wp-content/uploads/2020/03/SDCEPMADP-COVID-19-guide-300320.pdf (accessed October 2020).

3. Heggie C. Triaging of non-scheduled appointments in general dental practice: a clinical audit. Br Dent J 2019; 226: 203-206. 